\title{
DEVELOPMENT OF SANOFI PASTEUR TETRAVALENT DENGUE VACCINE
}

\author{
Jean LANG(1)
}

With almost $50 \%$ of the world population at risk and an estimated annual burden that includes $230 \mathrm{M}$ infections, $2 \mathrm{M}$ severe cases and 25000 deaths, dengue is a major public health concern. Brazil is a priority country for dengue vaccination as it accounts for $70 \%$ of all reported cases in Americas (1998-2009) and > 1 million clinical cases reported in 2010. After decades of research by different groups around the world, dengue vaccine development reached a major milestone in 2010 with the initiation of the first phase III clinical trial to investigate a candidate vaccine. This vaccine is the Sanofi Pasteur CYD tetravalent dengue vaccine (TDV) which is now being evaluated for protective efficacy in large scale trials in Latin America and south-east Asia as part of an extensive clinical development program in both regions. The CYD TDV candidate comprises four recombinant, live, attenuated vaccines (CYD-1-4) based on a yellow fever vaccine 17D (YF17D) backbone, each expressing the pre-membrane and envelope genes of one of the four dengue virus serotypes. The vaccine is genetically and phenotypically stable, non-hepatotropic, less neurovirulent than YF17D, and does not infect mosquitoes by the oral route. In vitro and in vivo preclinical studies showed that CYD TDV induces controlled stimulation of human dendritic cells, and significant immune responses in monkeys that crossreact against a panel of approximately 20 different circulating strains per serotype. Scale up and industrialization are being conducted in parallel with preclinical and clinical development to fulfill the needs of phase II/ III trials, and to anticipate and facilitate supply and access to vaccine in the countries where the dengue disease burden makes it an urgent public health priority. As of September 2011, the vaccine has been administered to more than 11,000 children and adults in completed or ongoing studies and no safety concerns have arisen. Given with a three-dose regimen, vaccination induces balanced immune responses against all four serotypes in the large majority of vaccinees. Preexisting flavivirus immunity favors quicker and higher immune responses to CYD TDV, without adversely effecting clinical safety or increasing vaccine viremia. The observed level and nature of the cellular immune responses in humans are consistent with the good safety and immunogenicity profile of the vaccine. Preliminary results of an ongoing efficacy and large scale safety study in Thai children are expected by the end of 2012 and we envisage the availability of this vaccine for high disease burden countries in the next three to five years.

The worldwide expansion of dengue fever is major public health concern. Dengue has become the most common tropical disease in Latin America and Asia Pacific, and worldwide dengue is second only to malaria. Almost $50 \%$ of the world population lives in areas at risk for dengue. Each year more than 230 million people are infected; two million patients develop severe disease, especially children (90\%), and 25,000 patients die ${ }^{1-3}$.

Dengue is spreading in different areas and the burden of disease has increased substantially in recent decades. Brazil accounts for the majority of cases registered in the Americas, with more than one million dengue cases, almost 100,000 hospitalizations and more than 600 deaths in $2010^{4}$.

There is no specific treatment against dengue and vector control measures have limited impact, particularly with the development of insecticide resistance among the mosquito population. The availability of a dengue vaccine would represent a major advance in the control of the disease $e^{2,4-6}$.

In the last 40 years, different approaches have been used by academic and industrial laboratories to develop dengue vaccine candidates. These include live attenuated virus (LAV), recombinant virus vectors expressing dengue envelope (E) antigens, purified inactivated virus (PIV), recombinant subunit vaccine, virus-like particles (VPLs), and DNA vaccines ${ }^{6-8}$.

Live vaccines have been shown to produce robust, long-lasting and broad humoral and cellular immune response, but are associated with a higher rate of adverse events, and cannot be used in some at-risk groups, such as in immunocompromised people. Inactivated vaccines have reduced potential for reactogenicity, but tend to have a diminished ability to induce broad and durable immune responses ${ }^{6}$.

Several dengue vaccines are at various stages of evaluation. The early enthusiasm about the tetravalent LAV dengue vaccines, developed with classic empirical techniques of attenuation by serial cell passages, was impacted by the difficulty of achieving an appropriate balance between immunogenicity and reactogenicity. The vaccine strains were genetically and phenotypically unstable, and caused denguelike syndromes (fever, leucopenia, and rash) in high proportion of participants in early clinical trials ${ }^{6}$. A second generation of dengue vaccines was developed to surmount these problems. In the early 2000's, Acambis discovery scientists (now Sanofi Pasteur) designed a second generation of flavivirus vaccines, using an innovative recombinant DNA technology to produce four live, attenuated vaccine viruses based on yellow fever 17D vaccine strain, one per dengue serotype. 
Each recombinant virus (CYD1, CYD2, CYD3 and CYD4) was constructed by removing the genes encoding the premembrane (prM) and envelope (E) proteins from the yellow fever 17D virus and inserting the corresponding genes from a dengue virus ${ }^{8}$. Using this technology, Sanofi Pasteur has preclinically, clinically and industrially developed a tetravalent dengue vaccine, combining the four CYD vaccine viruses into a single vaccine. This dengue candidate vaccine is the first to reach the milestone of clinical phase III studies ${ }^{8}$.

There are many hurdles to the development of a successful dengue vaccine, including:

- The lack of an animal model that reproduces human disease. Nonhuman primates can be infected but do not develop disease.

- Four dengue virus serotypes can cause serious diseases, and the immunity conferred by an infection is serotype-specific, with only a short period of cross-protection against infection by other serotypes.

- There is a theoretical risk of immunopotentiation or sensitization after sequential monovalent infections, which has been proposed to be caused, in part, by antibody dependent enhancement (ADE), associated with heterologous non-neutralizing antibodies and/or the waning of homologous neutralizing antibody titers.

- Neutralizing antibodies against the dengue envelop proteins are considered to be essential for protection, but there is no known correlate of protection, and candidate vaccines must be studied for efficacy in large numbers of individuals, in accordance with guidelines for the clinical evaluation, production and quality control recommended by WHO.

The ideal dengue vaccine would have a good safety profile, the ability to elicit broad and durable immune responses against all four serotypes, and be suitable for use in children and adults. Live vaccines usually confers long lasting protection, but the development of multivalent live vaccines is complicated by the potential for interference between serotypes which can result in the immunodominance of one or two serotypes, and can necessitate several doses to achieve an antibody response to all serotypes ${ }^{6,7}$.

Dengue affects people in all age groups. Virus circulation differs between regions, even within the same country. Vaccination schedules, including for other flaviviral diseases (yellow fever and Japanese encephalitis), differ between countries. How to fit a new vaccine into existing national immunization programs therefore requires careful consideration. It is therefore necessary to study the potential of interference of a dengue vaccine candidate when co-administered with established vaccines ${ }^{7,8}$.

Pragmatic issues, such as storage and the logistics of distributing a high number of doses while maintaining the cold-chain, and strategies to reach compliance with the recommended schedule and achieve suitable vaccine coverage rates require discussion with health authorities before implementation of a dengue vaccine ${ }^{9}$.

The effective use of the vaccine in the field will require partnerships and collaboration among public and private sectors of endemic countries to define where and how to introduce the vaccine. The ultimate goal will be to design effective vaccination programs to alleviate the growing global disease burden and pharmacoeconomic disruptive impact of dengue viruses ${ }^{2}$.
Other challenges relate to the industrialization of vaccine production for the four vaccine viruses, and the manufacturing process scale-up necessary to supply large-scale Phase III trials to demonstrate the protective efficacy, and to prepare for the subsequent launch in case of licensure ${ }^{7-10}$. Sanofi Pasteur has constructed new facilities to scale up the production, and facilitate supply and access to vaccine as soon as possible.

Extensive pre-clinical research has demonstrated the genotypic and phenotypic stability of the CYD vaccine strains and has shown that reversion to virulence or the recombination with other flaviviruses is highly unlikely. The risk of inadvertent environmental dissemination of the CYD viruses via mosquitoes has also been shown to be minimal ${ }^{7,8,11-13}$.

Other issue related to a candidate vaccine is its capability to protect against different genotypes of dengue, and we had shown that our candidate vaccine induces cross-reactive responses against a panel of about 20 different circulating strains per serotype ${ }^{8}$. "Cross-neutralizing antibody responses against circulating DENV field isolates after human vaccination with a tetravalent dengue vaccine ${ }^{14,15}$.

Phase I and II clinical trials with a 3-dose vaccination regimen over 12 months demonstrate that this candidate vaccine elicits neutralizing antibodies against all four serotypes in both children and adults with a good safety profile $\mathrm{e}^{13,16,17}$.

The global clinical development program includes 45,000 participants from 15 countries $^{8}$. To date (February 2012), more than 23,000 individuals, aged two to 45 years have received at least one dose of CYD dengue vaccine in completed or ongoing studies ${ }^{8}$. Vaccine safety is being monitored by an Independent Data Monitoring Committee (IDMC), an independent group of international experts, and so far no safety signals have been identified. A pooled analysis of safety data available in June 2011, including 1,728 subjects from 5 Phase I studies (USA, Mexico, Philippines) and eight phase II studies (six completed) also revealed no safety concerns. Reports of solicited reactions and unsolicited adverse events were similar to those of the control vaccines, and tend to decrease in frequency after second and third vaccinations compared to first vaccination. Vaccine viremia is undetectable in the large majority of vaccinees, and when detected the level of viremia has remained low. There have been no reports of dengue-like syndrome or serious adverse events related to this vaccine, in contrast to live attenuated vaccines developed by conventional attenuation techniques. Furthermore, the safety profile has been shown to be comparable in individuals with or without prior exposure to dengue of other flavivirus, via infection or vaccination (YF, $\mathrm{JE})^{7,8,14}$. While all available data show that the vaccine's safety profile is good, with no safety signals detected after the vaccination of more than 23,000 vaccinees so far, the potential risk of very rare adverse events cannot be determined until the licensure and introduction of the vaccine. Other questions that cannot be definitively answered until after vaccine introduction concern herd protection, efficacy of alternative schedules (intention to treat), and the duration of protection in endemic and nonendemic areas.

The first phase III study was initiated in 2010 in Australia to evaluate lot-to-lot consistency. The first efficacy study, including 4,000 children (4-11 years) is ongoing in a highly endemic area of Thailand, and the results are expected for the end of 2012. Positive results from this 
study, conducted in an area where the force of infection is high would represent the first time clinical efficacy has been demonstrated with a dengue vaccine, and would allow us to consider initial submission to the regulatory authorities in some countries ${ }^{8}$.

To expand the efficacy database in different countries, two other phase III efficacy studies are ongoing in Asia (Thailand, Indonesia, Malaysia, Viet Nan, Philippines), with planned inclusion of about 10,000 children (2-14 years) and Latin America (Colombia, Mexico, Honduras, Porto Rico and Brazil) where the number of enrolled children aged 9-16 years is expected to be 20,000 .

In conclusion, the CYD tetravalent dengue vaccine candidate has demonstrated its capacity to induce broad and balanced immune responses against the four dengue serotypes in children and adults from endemic and non-endemic areas with a 3 dose vaccination regimen at $0,6,12$ months. All available data show the vaccine's safety profile to be good, with no safety signals detected after the vaccination of more than 23,000 vaccinees so far. The outcome of the first dengue vaccine efficacy trial will be known by the end of 2012 and if positive will pave the way for initial licensure and the immunization of populations against this burdensome disease.

\section{REFERENCES}

1. World Health Organization. Dengue. [Cited: 13 Jan. 2012]. Available from: <http://www. who.int/>.

2. Amarasinghe A, Wichmann O, Margolis HS, Mahoney RT. Forecasting dengue vaccine demand in disease endemic and non-endemic countries. Hum Vaccin. 2010;6:745-53.

3. San Martin JL, Brathwaite O, Zambrano B, Solorzano JO, Bouckenooghe A, Dayan $\mathrm{GH}$, et al. The epidemiology of dengue in the Americas over the last three decades: a worrisome reality. Am J Trop Med Hyg. 2010;82:128-35.

4. Siqueira Jr JB, Vinhal LC, Said RFC, Hoffmann JL, Martins J, Barbiratto SB, et al. Dengue no Brasil: tendências e mudanças na epidemiologia, com ênfase nas epidemias de 2008 e 2010. Brasília: Portal da Saúde. [Cited: 20 Jan. 2010]. Available from:<www. saude.gov.br>.

5. Suaya JA, Shepard DS, Siqueira JB, Martelli CT, Lum LC, Tan LH, et al. Cost of dengue cases in eight countries in the Americas and Asia: a prospective study. Am J Trop Med Hyg. 2009;80:846-55.
6. Schmitz J, Roehrig J, Barrett A, Hombach J. Next generation dengue vaccines: a review of candidates in preclinical development. Vaccine. 2011;29:7276-84.

7. Guy B, Saville M, Lang J. Development of Sanofi Pasteur tetravalent dengue vaccine. Hum Vaccin. 2010;6:696-705.

8. Guy B, Barrere B, Malinowski C, Saville M, Teyssou R, Lang J. From esearch to phase III: preclinical, industrial and clinical development of the Sanofi Pasteur tetravalent dengue vaccine. Vaccine. 2011;29:7229-41.

9. Lam SK, Burke D, Capeding MR, Chong CK, Coudeville L, Farrar J, et al. Preparing for introduction of a dengue vaccine: recommendations from the $1^{\text {st }}$ Dengue v2V Asia-Pacific Meeting. Vaccine. 2011;29:9417-22.

10. Durbin AP, Whitehead SS. Dengue vaccine candidates in development. Curr Top Microbiol Immunol. 2010;338:129-43.

11. Guy B, Guirakhoo F, Barban V, Higgs S, Monath TP, Lang J. Preclinical and clinical development of YFV 17D-based chimeric vaccines against dengue, West Nile and Japanese encephalitis viruses. Vaccine. 2010;28:632-49.

12. Morrison D, Legg TJ, Billings CW, Forrat R, Yoksan S, Lang J. A novel tetravalent dengue vaccine is well tolerated and immunogenic against all 4 serotypes in flavivirus-naive adults. J Infect Dis. 2010;201:370-7.

13. Guy B, Almond J, Lang J. Dengue vaccine prospects: a step forward. Lancet. 2011;377(9763):381-2

14. Mantel N, Boaz M, Crevat D, Vial T, Grillet F, Lang J, et al. Cross-neutralizing antibody responses against circulating denv field isolates after human vaccination with a tetravalent dengue vaccine. American Society of Tropical Medicine and Hygiene; 2010. Am J Trop Med Hyg. 2011;85(6 Suppl):Abs.506. [60 th $^{\text {th }}$ Annual Meeting Abstract Book].

15. Barban V, Girerd Y, Aguirre M, Gulia S, Pétiard F, Riou P, et al. High stability of yellow fever 17D-204 vaccine: a 12-year restrospective analysis of large-scale production. Vaccine. 2007;25:2941-50.

16. Poo J, Galan F, Forrat R, Zambrano B, Lang J, Dayan GH. Live-attenuated tetravalent dengue vaccine in dengue-naïve children, adolescents, and adults in Mexico City: randomized controlled phase 1 trial of safety and immunogenicity. Pediatr Infect Dis J. 2011;30:A9-A10.

17. Capeding RZ, Luna IA, Bomasang E, Lupisan S, Lang J, Forrat R, et al. Live-attenuated, tetravalent dengue vaccine in children, adolescents and adults in a dengue endemic country: randomized controlled phase I trial in the Philippines. Vaccine. 2011;29:386372. 